\title{
Robot-Assisted Surgery
}

National Cancer Institute

\section{Source}

National Cancer Institute. Robot-Assisted Surgery. NCI Thesaurus. Code C140885.

A surgical technique that uses a specially designed robotic system to aid with the surgical procedure. 